\title{
Erratum to: Dispersive corrections in elastic electron-nucleus scattering: an investigation in the intermediate energy regime and their impact on the nuclear matter
}

\section{The Jefferson Lab Hall A Collaboration ${ }^{\star}$}

Published online: 10 September 2020

(c) Società Italiana di Fisica and Springer-Verlag GmbH Germany, part of Springer Nature 2020

Erratum to: Eur. Phys. J. A (2020) 56:126

https://doi.org/10.1140/epja/s10050-020-00135-7

In the original PDF online version of this article, the references $22-26$ were missing. These are the missing references.

\section{References}

22. A. Afanasev, P.G. Blunden, D. Hasell, B.A. Raue, Prog. Part. Nucl. Phys. 95, 245 (2017). arXiv:1703.03874

23. A. Aste, C. von Arx, D. Trautmann, Eur. Phys. J. A 26, 167 (2005). arXiv:nucl-th/0502074

24. J.L. Friar, M. Rosen, Ann. Phys. 87, 289 (1974)

25. M. Traini, Nucl. Phys. A 694, 325 (2001). arXiv:nucl-th/0103045

26. R. Gilman, D.W. Higinbotham, X. Jiang, A. Sarty, S. Strauch, LEDEX: Low energy deutron experiments E05-044 and E05-103 at Jefferson Lab (2015). http://hallaweb.jlab.org/experiment/LEDEX/ 


\section{The Jefferson Lab Hall A Collaboration}

P. Guèye ${ }^{1}$, A. A. Kabir ${ }^{2,3}$, P. Giuliani ${ }^{4}$, J. Glister ${ }^{5,6,7}$, B. W. Lee ${ }^{8}$, R. Gilman ${ }^{9}$, D. W. Higinbotham ${ }^{10}$, E. Piasetzky ${ }^{11}$, G. Ron ${ }^{11}$, A. J. Sarty ${ }^{5}$, S. Strauch ${ }^{12}$, A. Adeyemi ${ }^{3}$, K. Allada ${ }^{13}$, W. Armstrong ${ }^{15}$, J. Arrington ${ }^{15}$, H. Arenaövel ${ }^{16}$, A. Beck ${ }^{17}$, F. Benmokhtar ${ }^{18}$, B. L. Berman ${ }^{19}$, W. Boeglin ${ }^{20}$, E. Brash ${ }^{21}$, A. Camsonne ${ }^{10}$, J. Calarco ${ }^{22}$, J. P. Chen ${ }^{10}$, S. Choi $^{8}$, E. Chudakov ${ }^{10}$, L. Coman ${ }^{23}$, B. Craver ${ }^{23}$, F. Cusanno ${ }^{24}$, J. Dumas ${ }^{9}$, C. Dutta ${ }^{13}$, R. Feuerbach ${ }^{10}$, A. Freyberger ${ }^{10}$, S. Frullani ${ }^{24}$, F. Garibaldi ${ }^{24}$, J.-O. Hansen ${ }^{10}$, T. Holmstrom ${ }^{25}$, C. E. Hyde ${ }^{26,27}$, H. Ibrahim ${ }^{28}$, Y. Ilieva ${ }^{19}$, X. Jiang ${ }^{9}$, M. K. Jones ${ }^{10}$, A. T. Katramatou ${ }^{4}$, A. Kelleher ${ }^{29}$ ， E. Khrosinkova ${ }^{4}$, E. Kuchina ${ }^{9}$, G. Kumbartzki ${ }^{9}$, J. J. LeRose ${ }^{10}$, R. Lindgren ${ }^{24}$, P. Markowitz ${ }^{20}$, S. May-Tal Beck ${ }^{17}$, E. McCullough ${ }^{5,30}$, D. Meekins ${ }^{10}$, M. Meziane ${ }^{30}$, Z.-E. Meziani ${ }^{15}$, R. Michaels ${ }^{10}$, B. Moffit ${ }^{30}$, B. E. Norum ${ }^{23}$, G. G. Petratos ${ }^{4}$, Y. Oh ${ }^{8}$, M. Olson ${ }^{31}$, M. Paolone ${ }^{12}$, K. Paschke ${ }^{23}$, C. F. Perdrisat ${ }^{30}$, M. Potokar ${ }^{32}$, R. Pomatsalyuk ${ }^{10,33}$, I. Pomerantz ${ }^{11}$, A. Puckett ${ }^{34}$, V. Punjabi ${ }^{35}$, X. Qian ${ }^{36}$, Y. Qiang ${ }^{35}$, R. D. Ransome 9 , M. Reyhan ${ }^{9}$, J. Roche ${ }^{37}$, Y. Rousseau9 ${ }^{9}$, B. Sawatzky ${ }^{10}$, E. Schulte ${ }^{8}$, M. Schwamb ${ }^{16}$, M. Shabestari ${ }^{23}$, A. Shahinyan ${ }^{38}$, R. Shneor ${ }^{11}$, S. Širca ${ }^{39}$, K. Slifer ${ }^{23}$, P. Solvignon ${ }^{15}$, J. Song ${ }^{8}$, R. Sparks ${ }^{10}$, R. Subedi ${ }^{3}$, G. M. Urciuoli ${ }^{24}$,

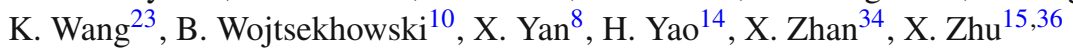

${ }^{1}$ National Superconducting Cyclotron Laboratory, Michigan State University, East Lansing, MI 48824, USA

${ }^{2}$ Hampton University, Hampton, VA 23668, USA

${ }^{3}$ Kent State University, Kent, OH 44242, USA

${ }^{4}$ Florida State University, Tallahassee, FL 32306, USA

${ }^{5}$ Saint Mary's University, Halifax, NS B3H 3C3, Canada

${ }^{6}$ Dalhousie University, Halifax, NS B3H 3J5, Canada

${ }^{7}$ Weizmann Institute of Science, Rehovot 76100, Israel

${ }^{8}$ Seoul National University, Seoul 151-747, Korea

${ }^{9}$ Rutgers, The State University of New Jersey, Piscataway, NJ 08855, USA

10 Thomas Jefferson National Accelerator Facility, Newport News, VA 23606, USA

${ }^{11}$ Tel Aviv University, Tel Aviv 69978, Israel

12 University of South Carolina, Columbia, SC 29208, USA

${ }^{13}$ University of Kentucky, Lexington, KY 40506, USA

14 Temple University, Philadelphia, PA 19122, USA

15 Argonne National Laboratory, Argonne, IL 60439, USA

${ }^{16}$ Institut für Kernphysik, Johannes Gutenberg-Universität, 55099 Mainz, Germany

${ }^{17}$ NRCN, P.O. Box 9001, Beer-Sheva 84190, Israel

${ }^{18}$ Duquesne University, Pittsburgh, PA 15282, USA

${ }^{19}$ George Washington University, Washington, D.C. 20052, USA

${ }^{20}$ Florida International University, Miami, FL 33199, USA

${ }^{21}$ Christopher Newport University, Newport News, VA 23606, USA

22 University of New Hampshire, Durham, NH 03824, USA

${ }^{23}$ University of Virginia, Charlottesville, VA 22094, USA

24 INFN, Sezione Sanitá and Istituto Superiore di Sanitá, Laboratorio di Fisica, 00161 Rome, Italy

${ }^{25}$ Longwood University, Farmville, VA 23909, USA

${ }^{26}$ Old Dominion University, Norfolk, VA 23508, USA

${ }^{27}$ Université Blaise Pascal / CNRS-IN2P3, 63177 Aubière, France

${ }^{28}$ Cairo University, Giza 12613, Egypt

${ }^{29}$ University of Western Ontario, London, Ontario N6A 3K7, Canada

${ }^{30}$ College of William and Mary, Williamsburg, VA 23187, USA

${ }^{31}$ Saint Norbert College, Greenbay, WI 54115, USA

32 Jožef Stefan Institute, 1000 Ljubljana, Slovenia

${ }^{33}$ NSC Kharkov Institute of Physics and Technology, Kharkov 61108, Ukraine

${ }^{34}$ Massachusetts Institute of Technology, Cambridge, MA 02139, USA

${ }^{35}$ Norfolk State University, Norfolk, VA 23504, USA

${ }^{36}$ Duke University, Durham, NC 27708, USA 
${ }^{37}$ Ohio University, Athens, OH 45701, USA

38 Yerevan Physics Institute, Yerevan 375036, Armenia

${ }^{39}$ Department of Physics, University of Ljubljana, 1000 Ljubljana, Slovenia 